\title{
Study on the toll plaza congestion based on game theory
}

\author{
ZHANG Gong-liang ${ }^{1}$ \\ School of management, \\ Guangdong University of Technology \\ Guangzhou, China \\ zhangdry1984@hotmail.com
}

\author{
CAO Ming ${ }^{2}$ \\ School of management, \\ Guangdong University of Technology \\ Guangzhou, China \\ caoming_ming@163.com
}

\author{
JIANG $\mathrm{Lu}^{3}$ \\ School of civil and transportation engineering \\ Guangdong University of Technology \\ Guangzhou, China \\ 944193048@qq.com \\ LI Wei-bin ${ }^{4}$ \\ Dongguan City Highway Bridge Development \\ and Construction Co \\ Dongguan, China \\ 273204629@qq.com
}

\begin{abstract}
The congestion of toll plaza seriously damages safety and smoothness of highway. In order to induce driver to reasonably choose charging channels, to improve the efficiency of toll station, and to relieve the congestion problem of highway toll plaza, this paper, considering drivers as the object of study and using evolutionary game approach, obtains two evolutionary stable strategies under different cost conditions by establishing game pay-off matrix, which can verify that there is equilibrium existing in the game of toll channel selection of drivers. Finally, some specific measures are proposed to balance the distribution of traffic flow of the toll plazas. Study shows that mixed strategy is equilibrium of game; to refine the functional area of toll plaza, to arrange quantity of charging channel reasonably and to allocate the vehicles in weaving section rationally are beneficial to induce strategic choices of drivers to be near the equilibrium, which efficiently relieves the congestion of the toll plazas.
\end{abstract}

Keywords-Highway transportation; Congestion of toll plaza; game theory; Selection of highway toll channels; Measures of balancing distribution

\section{INTRODUCTION}

With rapidly increasing of traffic and the development of the holiday tourism economy, The Intercity High Speed Road has gradually more crowded. There are so many vehicles in the large and medium-sized city with high population density places, especially in the rush hour. Traffic jams, occurred in the exit of highway toll station, had been a normal screen, and it has been seriously influence the Charging efficiency of the Operation enterprises and reduced the service function of highway.

Taking the Guangshen highway as example, at present, the average daily export traffic flow has been more than 400000 cars, and it had reached the saturation. In the rush hours, the export of high-speed channel of local road had more than the saturated state. If we lack of the correct induction for the car flow, the adding congestion phenomenon will further strengthen. Therefore, how to scientifically coordinate the toll plaza traffic with channel of charges and improve the efficiency of road has been the problem that had been studied by many scholars.

Game theory is widely applied to the study of traffic flow, Drik, German famous experts of traffic flow, combined the game theory with the study of traffic flow on many occasions [1] 。 Chinese scholars Hai-yan Xiao, Bing-quan liu (2009) combined with game distribution, proposed a new algorithm of traffic flow distribution and proved its validity and feasibility[2].

In the application of evolutionary game, with the help of evolutionary game analysis of drivers route choice behavior, Zhen-long Li ( 2003 ) divides the driver' into two types which are accepting induction and not accepting induction. according to established Evolutionary game model and simulated dynamic replication equation[3], Zhen-long Li clams that induced specification is very important for the driver's route choice[3]. Besides, Bo Zhang, Juan-zhi Cai \& An-ning Ni (2004) analyses the traveler route choice behavior of learning by evolutionary game[4]; through the establishment of dynamic traffic system model, it is proved that in satisfying certain conditions the system evolution can realize user equilibrium[4].

This article will take the driver as the research sample, and it assumed profits under different strategy, the driver's strategy is $s=\left(s_{1}, s_{2}, \cdots, s_{i}\right)$, $\xi(s)=\left\{\xi_{1}(s), \xi_{2}(s), \cdots, \xi_{n}(s)\right\}$, it is the payoff function (income) for each strategy[5]. The driver's payment is caused by themselves of different income and cost, the other people's participate will influence the self-choice. Therefore, the game relationship has appeared between the drivers.

There are $\mathrm{N}$ people partaking in the game. if $\xi_{i}=\left(s_{1}, s_{2}, \cdots, s_{n}\right)=\xi_{\eta(i)}^{-1}\left(s_{\eta(1)}, s_{\eta(2)}, \cdots, s_{\eta(n)}\right)$ exist, we call it symmetric game[5] [6], this article had used the evolutionary game theory to analyses the driver select charge channel behavior, and find the evolutionary equilibrium point, In order to help High speed road 
managers induced driver to choose reasonable charges channel. Through alleviate the toll plaza congestion problem; it can improve the high-speed road congestion.

\section{EVOLUTION MODEL}

In reality, toll plaza of highway has the plurality of charging channels, driver will usually have a variety of options, but because of the habits, the driver is usually prefer to choose in a directly way. The final results were the number of vehicles exceed the channel capacity and caused the traffic dam, and the driver needs to pay a high cost of time to wait for entering the service charges

Now the 6 toll lanes are divided into two categories, one is the Toll road channel straight connected with the vehicle, set it $\mathrm{A}$, and it is the shortest channel, more drivers are willing to choose this road, so it is full of cars, and caused traffic jam; the other kind of channel needs to be changed the way, and it is longer, set it $\mathrm{B}$, if driver choose this way, it will cost more driving costs(including the time cost, safety cost) to get to the destination.

Supposing that there are channel A and channel B , they all has the Operating limit and share the same traffic capacity. If all the drivers choose channel A or channel B, the traffic flow is greater than the channel capacity, it will caused the traffic jam, if the drivers do not willing to pay the driving cost, they all waiting in the channel A, for individuality, it save the driving cost, but it damaged the efficiency of the entire highway exit traffic channel.

All the drivers are the participants of the game, setting all the drivers on some period in one High speed road exit fees channel as $\mathrm{N}$ game participants, table 2 is the driver path selecting payment matrix, $\mathrm{w}$ is the participant's Initial return, a,k,g are respectively selecting appropriate channel cost payment. And it acquired the a, k, g>0. If all the drivers choose A, they all will pay the A's cost, if all the drivers choose $\mathrm{B}$, they will pay more $\mathrm{g}$ costs, including the cost and the cost of fuel.

When some drives choose channel A, and some other drivers choose channel $\mathrm{B}$, the drivers who choose the channel will not cost the external costs, so they will not exist the time cost, and will also cost the spare fuel, however the driver who choose channel $\mathrm{B}$ will cost more $\mathrm{k}$ costs.

TABLE I. THE PAYOFFS MATRIX OF ROUTES SELECTION OF DRIVERS

\begin{tabular}{llll}
\hline & & $\mathrm{A}$ & $\mathrm{B}$ \\
\hline$p$ & $\mathrm{~A}$ & $w-a$ & $w$ \\
$1-p$ & $\mathrm{~B}$ & $w-k$ & $w-a-g$ \\
\hline
\end{tabular}

The Driver group select strategy A with probability $\mathrm{p}$, with probability $1-\mathrm{p}$ select strategy B :

$$
E\left(S_{A}\right)=p(w-a)+(1-p) w
$$

The expected return to select strategy $\mathrm{B}$ is:

$$
E\left(S_{B}\right)=p(w-k)+(1-p)(w-a-g)
$$

Replicated dynamic equation by the payment matrix and the expected return to the game is:

$$
F(p)=\frac{d p}{d t}=p(1-p)\left[E\left(S_{A}\right)-E\left(S_{B}\right)\right]=p(1-p)[p(k-g-2 a)+a+g]
$$

Order $F(p)=0$, it can get Stable replication dynamic equation possible point:

$$
p_{1}=0, p_{2}=1, p^{*}=\frac{a+g}{2 a-k+g}
$$

Wherein, $0<p^{*}<1$, Parameters must satisfy $2 a-k+g>0 \quad ; \quad$ When $\quad p_{1}=0 \quad, \quad$ and $F^{\prime}(0)=a+g>0$

it is easy to figure that $p_{1}=0$ is not the evolutionary stable point ; when $p_{2}=1, \quad F^{\prime}(1)=a-g$; when $p^{*}=\frac{a+g}{2 a-k+g}, F^{\prime}\left(p^{*}\right)=\frac{(a-g)(a+g)}{2 a-k+g}$.

The following are the Discussion of weather $p_{2}$ 与 $\mathrm{p}^{*}$ is the evolution of the stable point :

(1) When $a-k<0,2 a-k+g>0, F^{\prime}(0)>0, F^{\prime}\left(p^{*}\right)>0, F^{\prime}(1)<0$, and $p_{2}=1$, it Is the evolution of the game equilibrium point, The replication dynamic equation of the trend graph as Fig .1 shows,

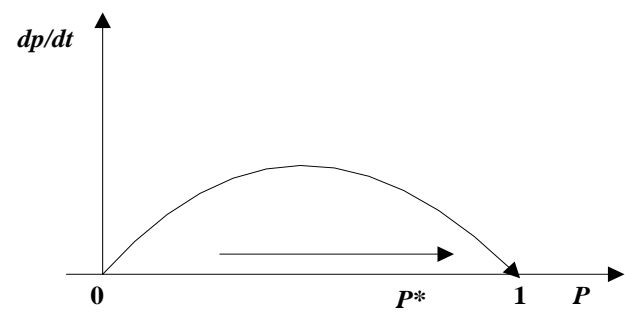

Figure 1. Replication dynamic trend figure when

$$
a-k<0,2 a-k+g>0 .
$$

Under these circumstances, strategy A have the shorter time and distance, the cost of strategy A will lower than strategy B, strategy B will be abandoned as the second-rate choice. All the driver will choose the strategy A to get the best benefit.

The total expected revenue for $\mathrm{N}$ drivers is $\mathrm{n}(\mathrm{w}-\mathrm{a})$, however, ( $\mathrm{A}, \mathrm{A})$, although it is the equilibrium strategy but it is not the best strategy, the driver pursue their own interests maximize the result, but it cannot maximize system benefits. The number of the number of vehicles on the A channel is greater than the optimal system, the channel is over used, the traffic efficiency is greatly reduced, and the drivers were trapped into the prisoner's dilemma .

(2) when $a-k>0,2 a-k+g>0$,

$F^{\prime}(0)>0, F^{\prime}(1)>0, F^{\prime}\left(p_{3}\right)<0, \quad p^{*}=\frac{a+g}{2 a-k+g}$ is the evolutionary equilibrium point of the game. The replication dynamic equation of the trend graph, as Fig .2 shows. 


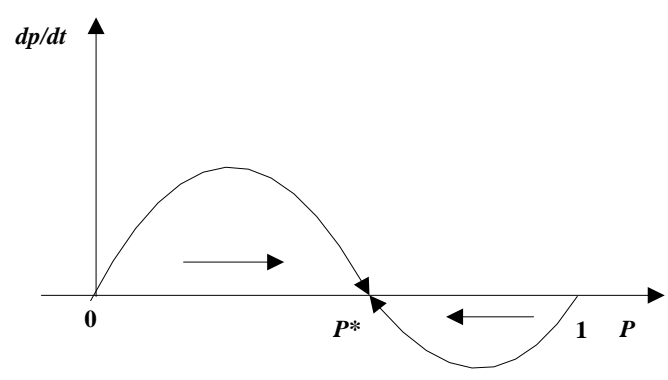

Figure 2. Replication dynamic trend figure when $a-k>0,2 a-k+g>0$

At the time, strategy A needs more time cost, and although the strategy B will take a longer distance, but it saved port of the waiting time, Select strategy A will cost more than the cost of select strategy B. only $\mathrm{g}>0$, no matter $\mathrm{g}$ is what value, it will not influence the evolution results. And when all the drivers had selected the strategy B, it will get the lowest proceeds, when the driver group using the $\frac{a+g}{2 a-k+g}$ probability to select the strategy A. and using the $\frac{a-g}{2 a-k+g}$ probability to select strategy $\mathrm{B}$, the game will reach the evolutionary stable state.

The toll plaza is for driver to choice game channel's venues; distribution state of the game is the result of traffic when the driver selection excessively focused on strategy A or strategy B. the traffic excessively was focused on one of the channel, it is not the best optimal steady state, this article analyses the driver selecting the charge channel behavior, its aim is to make the toll plaza traffic flow to achieve balanced. And making the charge peak reached the game Steady state.

\section{THE MEASURES OF BALANCING TRAFFIC FLOW DISTRIBUTION}

Through the analysis of the driver selecting the charging channel based on evolution game, there is a equilibrium point existed on the distribution of traffic at the toll plazas. Fig .3 is a non-equilibrium distribution of traffic at the toll plazas. The traffic of the charging channels that are near to the mainline first lane is greater than that of others. This phenomenon leads to the charging efficiency is poor.

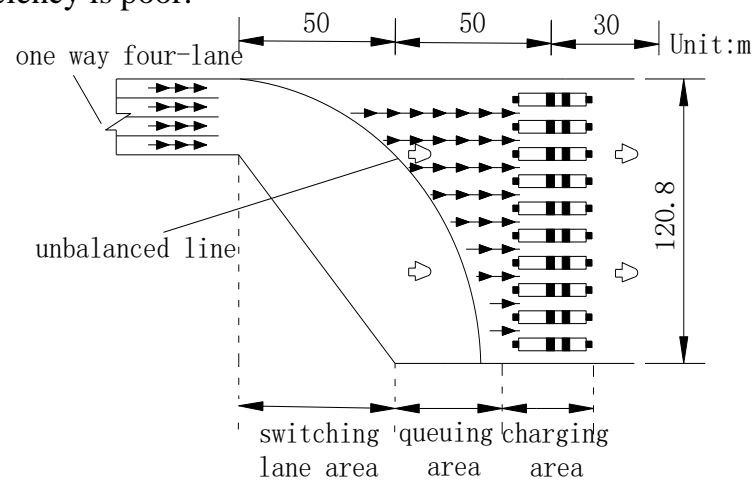

Figure 3. Distribution of traffic at the toll plazas of non-equilibrium State
In order to achieve an equilibrium distribution of traffic flow, some recommends measures can be adopted as following :

\section{A. Refined the domain of toll plaza}

As a perfect toll plaza, the design of domain should include: Widened area at the entrance of the plaza, weaving areas for Vehicle, waiting zone for queuing of vehicles, service area for charging, and so on[7]. The Vertical Section of each area above are different, but the traffic of Vertical Section must be equal[8]. otherwise, traffic Jam and Overflow might occur on the toll plaza. Therefore, reasonable partition of toll plaza, better designing of length and width of partitions, and reasonable number of lanes are the conditions for Achieving the balance of traffic at the toll plazas.

\section{B. Reasonable arrangements for the quantity of charging channel}

The amount $(\mathrm{n})$ of Charging channels for public can be calculated by:

$$
\mathrm{n} \geq \frac{t_{21}}{t_{11}}
$$

wherein: $t_{11}$ - Average headway of mainline lanes for a certain vehicle

$t_{21}$ - The average service time of the same type vehicles at the charging channels

The amount of opening charging channels is the ratio between he average service time of the same type vehicles at the charging channels and Average headway of mainline lanes[9]. Because the exclusive service time of charging channel is faster than mixed service. Therefore, to siphon off the traffics by large, medium and small vehicles in Main line driveway, the entering Vehicles of the plaza lining up by different class, to avoid different type of car mixed cut, And to provide adequate charging channel, are the measures for achieving the balanced distribution of traffic at the toll plazas.

\section{C. rational allocation of vehicles weaving sections}

vehicles entering the toll plaza From mainline, the road cross section changes. drivers in the face of the square, tend to choose the nearest charging channel for queue[10]. For waiting is not evenly distributed, vehicles inevitably appear the phenomenon of wear left and right. In order to provide a chance for drivers to choose ideal charging channel when they are entering the plaza, a vehicle weaving areas shall be set up at toll plaza.

length of weaving section is calculated as follows:

$$
L_{B}=\frac{V_{2}^{2}-V_{1}^{2}}{26 a}
$$

wherein: $L_{B}-$ length of weaving segment of toll Plaza;

$$
\begin{aligned}
V_{1}, V_{2}- & \text { the initial velocity and terminal velocity of } \\
& \text { Vehicles entering the toll plaza } \\
& \text { respectively }
\end{aligned}
$$




$$
a \text { - Reduction in vehicle acceleration, take 2-3 }
$$
$\mathrm{m} / \mathrm{s}^{2}$

At a setting speed, a car lane-permissible combination number is :

$$
n_{1}=\frac{L_{B} \operatorname{tg} \frac{\alpha}{2}}{b}-1
$$

wherein: $\alpha$ - Interwoven vehicle safety angle

$$
\text { is } 20^{\circ}-30^{\circ} \text {; }
$$

B- the width of one lane;

$n_{1}$ - number of Weaving lane.

(10), (11) showed that a vehicle entering the square with speed $V_{1}$, After decelerating the accelerating on $n_{1}$ security lines, enters a temporary waiting area with speed $V_{2}$.In other words, Set length of interlaced section $L_{B}$, Vehicles can be safely select $n_{1}$ Channel to line up. Weaving sections of toll plazas is the game zone for vehicle adjusting choice premium channel, In order to make the game evolution equilibrium, the More charging channel you set, longer reasonable weaving section provided by Plaza. Interwoven does not meet the length of the square, steps should be taken, allows drivers to traffic control personnel and traffic signs under the guidance of a slow, safely reached pay channel.

\section{CONCLUSION}

The article is focused on the driver's behavior for highway toll channel selection; through the establish of the payoff matrix, and using the evolutionary game theory to achie the game's expected revenue and replicate dynamics equation, the research get the different circumstances of the evolution phase diagram and dynamic evolution diagram.

Through the analysis we can get that in the toll channel selection game, there exists the equantequation, and the evolution path of equilibrium point are related to the initial value and driving cost. It will be close to the equilibrium point which was starting from different initial values. In order to improve the traffic efficiency of toll road, the road manager can take the refinement for toll square function area, and keeps a reasonable arrangements for the charge number of channels, taking a rational allocation of square weaving segment vehicle measure to guide the driver groups close the equilibrium point, and making traffic flow of each charge channel distribution reasonably, then it can improve the problem of toll plaza congestion.

\section{ACKNOWLEDGMENT}

I would like to show my deepest gratitude to the sponsors. This work was supported in part by the National Science Foundation of China with grant No. 71171061; and Guangdong Provincial Communications Department Science and technology project with grant No. 502140002 .

\section{REFERENCES}

[1] H. Drik "Pattern rormation,social forces, and diffusion instability in gamea with success-driven motion," The European Journal B London, vol. 67, pp.345-356. April 2009

[2] H. Xiao, B. Liu. "A new traffic assignment algorithm based on the shortest paths and game theory".Journal of south China normal university.China, vol. 1.pp.34-38. May 2009

[3] Z. Li, "A study of route choice behavior of drivers based on the evolutionary game under the condition of traffic flow guidance,"Journal of transportation systems engineering and information technology, China,vol.3,pp.23-27. May 2003

[4] B. Zhang, Z. Juan, and A. Ni. "An Evolutionary game model for the dynamic traffic flow based on cumulative prospect theory," Journal of Industrial Engineering,China,vol.3, pp. 164-173. 2014

[5] J. Smith. "Evolution and the Theory of Games." Cambridge university press, 1982 .

[6] G. Zhang, C. Zhang, H. Zhu "Linear Quadratic Zero-Sum Differential Games for a Class of Linear Systems with Markov Jump Parameters," Proceedings of the 5th International Asia Conference on Industrial Engineering and Management Innovation.Atlantis Press, China, vol.33. pp.161-164. 2015

[7] Z. Ding, X. Liu, and L.Tian "ESS, NIS and GIS for multi - player matrix game in single population," Mathematical Methods in the Applied Sciences.China,vol.33.pp.332-339. 2010,

[8] D. ZHANG, X. TAN.'Simulation research of vehicles' queuing system in toll plaza".Journal of system simulation. China, vol.18.pp.1065-1069. 2006.

[9] F. Hajiseyedjavadi, I. McKinnon C. Fitzpatrick, "Application of Microsimulation to Model the Safety of Varied Lane Configurations at Toll Plazas," Transportation Research Board 94th Annual Meeting. No.(15-3480).2015.

[10] L. Zhong, Y Zhou, K. Wu,"Analysis of Level of Service of Toll Lane Allocation of Hong Kong-Zhuhai-Macao Bridge," Safe, Smart, and Sustainable Multimodal Transportation Systems. ASCE,Vol.a11.pp.1863-1870. 2014 